\title{
Uso de plasma rico em plaquetas intra-articulares como tratamento pós-cirúrgico da ruptura do ligamento cruzado cranial num cão
}

\author{
[The use of intra-articular platelet rich-plasma as a postsurgical treatment of cranial cruciate \\ ligament rupture in a dog] \\ R.F. Silva ${ }^{1,2}$, J.U. Carmona ${ }^{2}$, C.M.F. Rezende ${ }^{1}$ \\ ${ }^{1}$ Escola de Veterinária - Universidade Federal de Minas Gerais - Belo Horizonte, MG \\ ${ }^{2}$ Grupo Terapia Regenerativa - Universidad de Caldas, Manizales, Caldas, Colombia
}

\begin{abstract}
RESUMO
Relata-se o caso de um cão que recebeu injeções intra-articulares de plasma rico em plaquetas (PRP) durante o pós-operatório do tratamento cirúrgico de ruptura do ligamento cruzado cranial (RLCCr). Os resultados clínicos e da avaliação da marcha mediante plataforma de força neste paciente sugerem a utilização de injeções intra-articulares de PRP como terapia pós-cirúrgica no tratamento da RLCCr.
\end{abstract}

Palavras-chave: cão, ligamento cruzado cranial, plasma rico em plaquetas

\begin{abstract}
We report the case of a dog that received intra-articular injections of platelet-rich plasma (PRP) during the postoperative period of surgical treatment of cranial cruciate ligament (CCL) rupture. Clinical, and gait analysis by the force plate in this patient might suggest the use of intra-articular injections of PRP as a post-surgical therapy in the treatment of CCL rupture.
\end{abstract}

Keywords: dog, cranial cruciate ligament, platelet rich plasma

\section{INTRODUÇÃO}

A ruptura do ligamento cruzado cranial (RLCCr) é uma das principais causas de claudicação no cão (Vasseur, 2003). O tratamento cirúrgico inclui várias técnicas baseadas em estabilização extracapsular e intracapsular, e osteotomia de nivelamento do platô tibial (TPLO) (Conzemius et al., 2005). Independentemente da técnica utilizada, as maiores complicações pós-cirúrgicas são a diminuição da funcionalidade e progressão da osteoartrose (OA) da articulação fêmorotíbio-patelar (Elkins et al., 1991; Hurley et al., 2007). O plasma rico em plaquetas (PRP) é um concentrado natural de fatores de crescimento, principalmente fator de crescimento transformador $\beta$ (TGF- $\beta$ ), fator de crescimento insulínico (IGF), fator de crescimento derivado das plaquetas (PDGF) e outras proteínas que modulam a inflamação e a cicatrização (Anitua et al., 2007). Resultados preliminares indicam que o tratamento com injeções intra-articulares (IA) de PRP é seguro e tem o potencial para reduzir a dor e melhorar a funcionalidade da articulação fêmoro-tíbio-patelar e a qualidade de vida em pacientes humanos com degeneração articular (Kon et al., 2009; Filardo et al., 2010). Existem relatos em medicina veterinária sobre a utilização IA de PRP no tratamento de OA em cavalos (Carmona et al., 2007) e de osteocondrite dissecante da articulação escapuloumeral em um cão (Neumann e Viefhues, 2010). O objetivo deste relato é descrever o caso de um cão com RLCCr tratado cirurgicamente por artroscopia para substituir o ligamento cruzado cranial (LCCr) por enxerto autólogo de fáscia lata e que recebeu terapia póscirúrgica com PRP obtido mediante o método do tubo (Silva et al., 2011). O fato relevante deste relato é que, após a aplicação de PRP,

Recebido em 2 de março de 2011

Aceito em 22 de maio de 2012

E-mail: raul.silva@ucaldas.edu.co 
observou-se rápido retorno da funcionalidade do membro e mínima progressão das alterações radiográficas de $\mathrm{OA}$ em comparação com controles históricos.

\section{CASUÍSTICA}

Foi avaliado um cão macho da raça Boxer de um ano de idade e $23 \mathrm{~kg}$ de massa corporal, com deambulação bamboleante e claudicação acentuada do membro posterior direito com 10 dias de duração, posterior à queda em um buraco. Ao exame clínico, verificou-se teste de compressão tibial positivo, bem como o deslocamento cranial da tíbia em relação ao fêmur (gaveta cranial). $\mathrm{Na}$ artroscopia para avaliação das condições articulares, foi confirmado diagnóstico de RLCCr e realizado tratamento cirúrgico mediante substituição do LCCr por autoenxerto de fáscia lata (Muzzi et al., 2009). O paciente recebeu carprofeno $(2,2 \mathrm{mg} / \mathrm{kg})$ e cefalexina $(30 \mathrm{mg} / \mathrm{kg})$ a cada 12 horas, ambos por via oral, durante sete dias. O membro foi imobilizado, durante 15 dias, com muleta de Thomas modificada. Foram feitas três injeções IA de PRP como terapia pós-cirúrgica em intervalos de 15 dias. O paciente foi avaliado radiograficamente para determinar a evolução do processo degenerativo e foi também submetido à avaliação objetiva da marcha em plataforma de força mediante determinação das forças de apoio vertical para cada membro.

A avaliação radiográfica foi feita antes da cirurgia e aos 15, 30, 60 e 90 dias de póscirúrgico. A articulação fêmoro-tíbio-patelar foi avaliada nas posições mediolateral e craniocaudal. Os sinais radiográficos de $\mathrm{OA}$ foram analisados por meio da escala de Innes et al. (2004), na qual se avaliam quatro parâmetros: efusão articular (0-2), osteofitose (0-3), mineralização intra-articular (0-2) e esclerose subcondral tibial (0-1). A pontuação total é obtida mediante a soma e a média dos valores de cada parâmetro. Neste sentido 0 é normal e 3 é OA grave.

A avaliação da marcha para determinar o grau objetivo de claudicação e, indiretamente, da dor foi feita em plataforma de força com um sistema de aquisição de dados NI-DAQ ${ }^{\mathrm{TM}}$ (National Instruments, USA), colocada numa pista de marcha de três metros de comprimento por $50 \mathrm{~cm}$ de largura e $10 \mathrm{~cm}$ de altura. As forças verticais de reação do solo que atuam entre o membro e a plataforma foram avaliadas na fase de apoio do ciclo de marcha. O paciente caminhou no seu

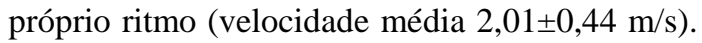
A velocidade da marcha foi calculada com base no tempo de ciclo da extremidade anterior esquerda, pela fórmula: velocidade $=$ frequência (ciclos/s) x amplitude (m) (Bertram e Ruina, 2001), para o qual se utilizou uma videocâmera a $60 \mathrm{~Hz}$ (SONY DCR-SX63, China). Avaliaram-se três ciclos válidos para cada membro. Considerou-se como ciclo válido o apoio ipsilateral dos membros. Os dados foram adquiridos a uma velocidade de $300 \mathrm{~Hz}$ e processados no programa DasyLab 10 (Measurement Computing, USA). Os dados avaliados foram o pico de força vertical $\left(\mathrm{Pf}_{\mathrm{z}}\right)$ e o impulso vertical $\left(\mathrm{If}_{\mathrm{z}}\right)$, que foram normalizados à massa corporal (N/kg e N/kg/s, respectivamente). Cada variável foi expressa em porcentagem do total da força-impulso exercida por todos os membros mediante o uso da fórmula: $\mathrm{X} \%_{\text {(membro }}$ A) $=\mathrm{F}_{\text {(membro }}$ A) $/\left(\mathrm{F}_{\text {(membro }} \mathrm{A}\right)+\mathrm{F}_{\text {(membro }}$ B) $+\mathrm{F}_{\text {(membro }}$ C) $+\mathrm{F}_{\text {(membro D) }}$ ) (Katic et al., 2009), em que: X\% representa a porcentagem de massa corporal distribuída através do membro; A, B, C e D representam os quatro membros do cão; e F representa o valor de força-impulso do membro correspondente. Calculou-se a média do valor dos três ciclos de marcha por membro para a normalização das variáveis e utilizou-se este valor para as análises. As análises cinéticas foram feitas nos mesmos tempos que as avaliações radiográficas.

O PRP foi preparado de maneira asséptica, segundo a técnica descrita por Silva et al. (2011). Obteve-se o sangue por meio da venopunção safena com scalp $21 \mathrm{G}$ (Shandong Weigao Group, China), o qual foi depositado em dois tubos de $10 \mathrm{~mL}$ contendo $1,5 \mathrm{~mL}$ de solução A de ACD (BD, New Jersey, USA). O sangue foi centrifugado a 191g (aceleração da gravidade) durante seis minutos. Com auxílio de uma agulha espinal 18G, coletou-se $1 \mathrm{~mL}$ de plasma adjacente à interface hemácias-plasma. O PRP foi analisado mediante hemograma automatizado (Abacus Junior Vet, Austria) e comparado com os valores celulares do sangue total. Foram aplicadas três doses de $1,5 \mathrm{~mL}$ de PRP via IA, sendo a primeira imediatamente após o procedimento cirúrgico e as outras duas em intervalo de duas semanas. O PRP foi ativado com gliconato de cálcio $(100 \mathrm{mg} / \mathrm{mL})$ 
(Hipolabor $^{\circledR}$, Belo Horizonte, Brasil) na relação 10:1 imediatamente antes de ser injetado na articulação.

\section{DISCUSSÃO E CONCLUSÃO}

O paciente não mostrou complicação sistêmica ou local associada com o procedimento cirúrgico ou com a aplicação IA de PRP. A contagem média de plaquetas $(514,050 \pm 145,119$ plaquetas $/ \mu \mathrm{L}$ ) no PRP injetado foi superior à contagem de plaquetas no sangue total $(376,333 \pm 45,457$ plaquetas $/ \mu \mathrm{L})$. A contagem de leucócitos no sangue total $(12,071 \pm 8018$ células $/ \mu \mathrm{L}$ ) em relação ao número de leucócitos presentes no PRP $(12,367 \pm 2303$ células/ $\mu \mathrm{L})$ foi similar. Neste último, as principais células encontradas foram linfócitos (61\%) e granulócitos (35\%). Estes valores são semelhantes aos resultados obtidos em concentrado de plaquetas de cão por meio do método do tubo (Silva et al., 2011).

Ao exame radiográfico pré-cirúrgico, detectou-se um defeito osteocondral na área de inserção tibial do LCCr (área intercondiloide cranial da tíbia), o qual se mostrou reduzido na avaliação aos 90 dias (Fig. 1). Na avaliação radiográfica mediante a escala de Innes et al. (2004), os valores foram semelhantes àqueles observados antes da cirurgia, aos 15, 30, 60 e 90 dias de pós-operatório. A esclerose subcondral tibial foi observada desde o período pré-cirúrgico, mostrando aspecto radiográfico mais acentuado aos 90 dias. Os demais parâmetros radiográficos apresentaram pontuação zero em todos os momentos de avaliação.

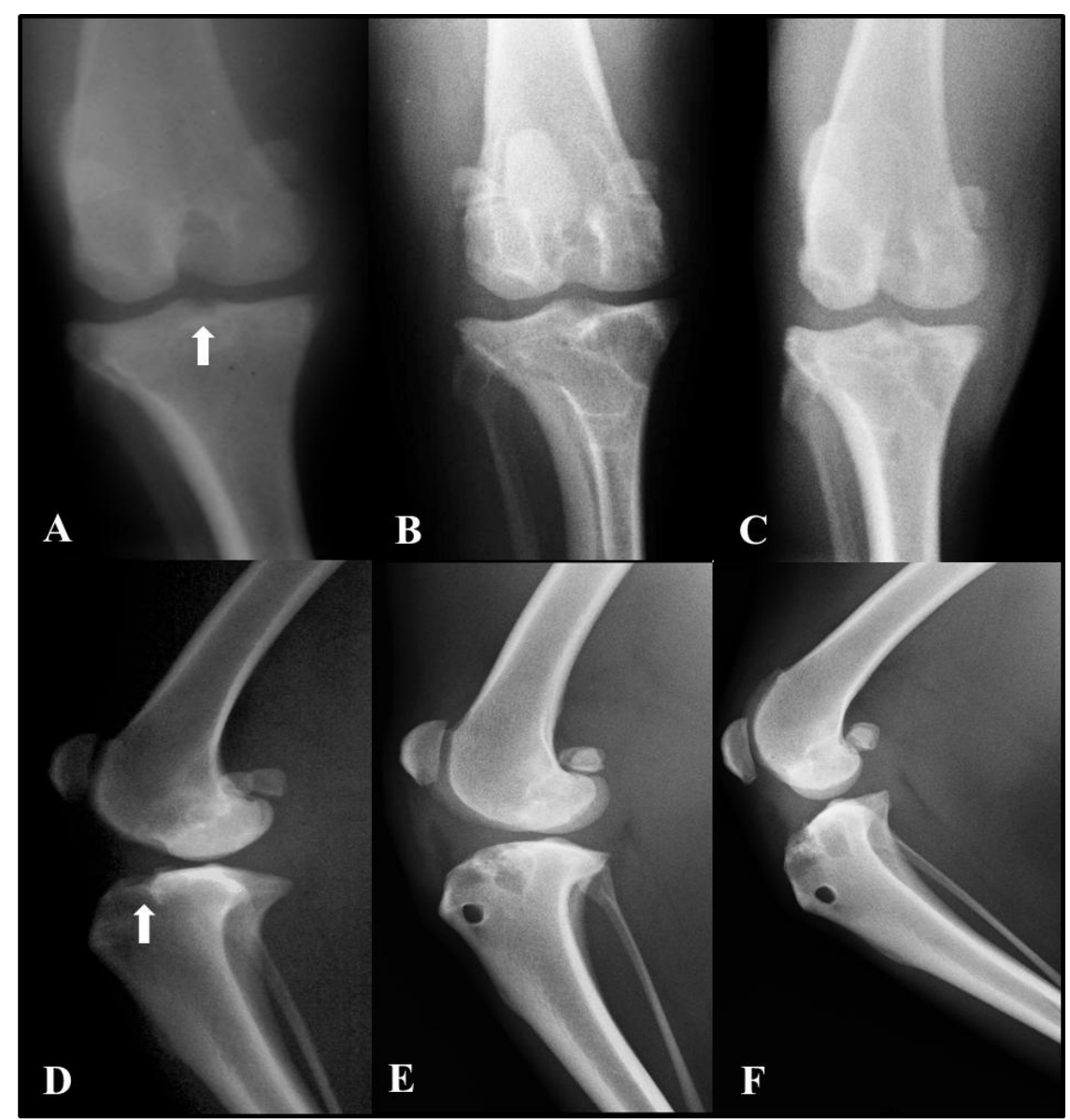

$\uparrow$ Defeito osteocondral

Figura1. Radiografias craniocaudal (A, B, C) e mediolateral (D, E, F) de cão submetido a tratamento cirúrgico da ruptura do ligamento cruzado cranial associado a injeções intra-articulares de plasma rico em plaquetas (PRP), antes (A, D) e aos 60 (B, E) e 90 (C e F) dias pós-operatórios. 
A avaliação da locomoção na plataforma de força indicou que os valores de pico de força vertical $\left(\mathrm{Pf}_{\mathrm{z}}\right)$ e de impulso vertical $\left(\mathrm{If}_{\mathrm{z}}\right)$ mostravam melhora gradual da funcionalidade do membro operado a partir do $15^{\circ}$ dia (Tab. 1). Antes da cirurgia, a distribuição percentual de apoio relativo aos valores de $\mathrm{Pf}_{\mathrm{z}}$ foi de $61.31 \%$ nos membros anteriores e $20,60 \%$ no membro acometido, devido à deambulação bamboleante que $\mathrm{o}$ paciente apresentava. $\mathrm{O}$ apoio entre membros contralaterais mostrou valores semelhantes na distribuição do peso a partir do dia 30 (Tab. 1).

Tabela 1. Porcentagem da distribuição da força aplicada nos quatro membros de cão representada pelo pico de força vertical $\left(\mathrm{Pf}_{\mathrm{z}}\right)$ e impulso vertical $\left(\mathrm{If}_{\mathrm{z}}\right)$, antes do tratamento cirúrgico de ruptura de ligamento cruzado cranial associado a injeções intra-articulares de plasma rico em plaquetas (PRP) aos 15, 30, 60 e 90 dias do pós-operatório

\begin{tabular}{cccccc}
\hline Variável & Tempo (dias) & MPD* \% & MPE \% & MAD \% & MAE \% \\
\hline \multirow{3}{*}{$\mathrm{Pf}_{\mathrm{z}}$} & 0 & 20,60 & 18,09 & 31,65 & 29,66 \\
& 15 & 17,98 & 20,02 & 31,11 & 30,89 \\
& 30 & 18,51 & 18,69 & 30,94 & 31,86 \\
& 60 & 18,96 & 19,17 & 31,26 & 30,61 \\
If $_{\mathrm{z}}$ & 90 & 19,41 & 19,29 & 30,24 & 31,06 \\
\hline & 15 & 16,59 & 18,52 & 33,56 & 31,33 \\
& 30 & 17,12 & 17,99 & 32,18 & 32,71 \\
& 60 & 19,09 & 19,53 & 30,09 & 31,29 \\
& 90 & 18,72 & 18,68 & 30,68 & 31,92 \\
\end{tabular}

MPD: membro posterior direito, MPE: membro posterior esquerdo, MAD: membro anterior direito, MAE: membro anterior esquerdo. *Membro tratado.

Os resultados clínicos e cinéticos mostram, neste caso, efeitos favoráveis do PRP com relação ao retorno funcional precoce do membro, mas são necessários estudos continuados para a avaliação de seu efeito em um número maior de casos clínicos. A relevância clínica deste relato é noticiar o emprego de PRP como um tratamento adicional à estabilização da articulação fêmorotíbio-patelar, de fácil execução, autólogo e com provável efeito regulador da $\mathrm{OA}$, devido à ação dos fatores de crescimento contidos no PRP.

Estudos feitos em cães com RLCCr tratados mediante técnicas cirúrgicas intra-capsulares têm reportado evidência radiográfica de OA a partir das quatro semanas (Elkins et al., 1991). Hurley et al. (2007) encontraram evidência radiográfica de OA da articulação fêmoro-tíbio-patelar oito semanas após a realização de TPLO. Vale ressaltar, entretanto, que, além das diferentes condições articulares em casos clínicos, nessa pesquisa de Hurley et al. (2007) só foram realizadas duas radiografias, uma antes do tratamento cirúrgico e outra oito semanas depois, não se podendo descartar a possibilidade de sinais radiográficos de $\mathrm{OA}$ antes da oitava semana. Segundo experimento de Muzzi et al. (2009) utilizando a técnica de reparação artroscópica, observam-se alterações compatíveis com $\mathrm{AO}$ já às quatro semanas pós-operatórias. $\mathrm{O}$ retorno funcional precoce no cão deste relato de caso, associado à discreta evolução dos achados radiográficos, sugere ação benéfica do PRP no controle da evolução do processo degenerativo de OA, pelo menos no período estudado.

Tanto a avaliação clínica (subjetiva) como a cinética (objetiva) do grau de claudicação mostraram melhora gradual da locomoção do paciente já aos 15 dias, ausência de claudicação aos 30 e aos 90 dias, período final de avaliação. Os resultados observados neste cão são promissores, já que pacientes com RLCCr tratados com TPLO, independentemente de receberem ou não fisioterapia pós-operatória, podem apresentar claudicação até seis semanas pós-cirurgia (Monk et al., 2006). Trumble et al. (2005) reportaram diminuição acentuada do $\mathrm{Pf}_{\mathrm{z}}$ duas semanas depois do tratamento cirúrgico extracapsular da RLCCr, resultado oposto ao encontrado neste relato de caso. Outro indício que sugere o papel modulador da dor e da inflamação pelo PRP empregado como terapia IA no paciente deste relato é o fato de que cães operados com a mesma técnica (com ou sem fisioterapia pós-operatória) não mostraram 
melhora dos parâmetros cinéticos ao término de quatro meses pós-operatórios (Muzzi et al., 2009).

Ainda é discutido e pouco se conhece o mecanismo do PRP na articulação. Existem relatos de que o TGF- $\beta$ possui efeito antiinflamatório e promove a diferenciação de células-tronco sinoviais em cartilagem (Pei et al., 2009). Estudos in vitro demonstram que o PRP incrementa a produção de ácido hialurônico em sinoviócitos humanos (Anitua et al., 2007). Estudos histológicos e testes de resistência biomecânica em caninos (Murray et al., 2006; Murray et al., 2007) concluem que a adição de PRP a um biomaterial de colágeno estimula a cicatrização do LCCr. Portanto, o PRP teria múltiplas ações nos tecidos moles e duros de articulações com OA secundária à RLCCr. Os resultados obtidos no paciente deste relato de caso sugerem o possível potencial do PRP como alternativa biológica, versátil e econômica. A ação moduladora do PRP sobre a OA já foi relatada em cavalos com AO que receberam tratamento intra-articular com PRP (Carmona et al., 2007). É necessário, portanto, realizar pesquisas duplo-cego aleatórias e controladas para demonstrar o potencial terapêutico desta substância como terapia pós-cirúrgica no tratamento da RLCCr em cães.

\section{AGRADECIMENTOS}

Os autores agradecem à Coordenação de Aperfeiçoamento de Pessoal de Nível Superior (CAPES) e à Fundação de Amparo à Pesquisa do Estado de Minas Gerais (FAPEMIG).

\section{REFERÊNCIAS}

ANITUA, E.; SÁNCHEZ, M.; NURDEN, A.T. et al. Platelet-released growth factors enhance the secretion of hyaluronic acid and induce hepatocyte growth factor production by synovial fibroblasts from arthritic patients. Rheumatology, v.46, p.1769-1772, 2007.

BERTRAM, J.E.A.; RUINA, A. Multiple walking speed-frequency relations are predicted by constrained optimization. J. theor. Biol., v.209, p.445-453, 2001.

CARMONA, J.U.; ARGÜELLES, D.; CLIMENT, F. et al. Autologous platelet concentrates as a treatment of horses with osteoarthritis: a preliminary pilot clinical study. J. Equine Vet. Sci., v.27, p.167-170, 2007.
CONZEMIUS, M.G.; EVANS, R.B.; BESANCON, M.F. et al. Effect of surgical technique on limb function after surgery for rupture of the cranial cruciate ligament in dogs. J. Am. Vet. Med. Assoc., v.226, p.232-236, 2005.

ELKINS A.D.; PECHMAN, R.; KEARNEY, M.T. et al. A retrospective study evaluating the degree of degenerative joint disease in the stifle joint of dogs following surgical repair of anterior cruciate ligament rupture. J. Am. Anim. Hosp. Assoc., v.27, p.533-540, 1991.

FILARDO, G.; KON, E.; BUDA, R. et al. Platelet-rich plasma intra-articular knee injections for the treatment of degenerative cartilage lesions and osteoarthritis. Knee. Surg. Sports. Traumatol. Arthrosc., v.19, p.528535,2010

HURLEY, C.R.; HAMMER, D.L.; SHOTT, S. Progression of radiographic evidence of osteoarthritis following tibial plateau leveling osteotomy in dogs with cranial cruciate ligament rupture: 295 cases (2001-2005). J. Am. Vet. Med. Assoc., v.230, p.16741679,2007

INNES, J.F.; COSTELLO, M.; BARR, J.F. et al. Radiographic progression of osteoarthritis of the canine stifle joint: a prospective study. Vet. Radiol. Ultrasound., v.45, p.143-148, 2004.

KATIC, N.; BOCKSTAHLER, B.A.; MUELLER, M. et al. Fourier analysis of vertical ground reaction forces in dogs with unilateral hind limb lameness caused by degenerative disease of the hip jointand in dogs without lameness. Am. J. Vet. Res., v.70, p.118126, 2009.

KON, E.; BUDA, R.; FILARDO, G. et al. Platelet-rich plasma: intra-articular knee injections produced favorable results on degenerative cartilage lesions. Knee. Surg. Sports. Traumatol. Arthrosc., v.18, p.472479, 2009.

MONK, M.L.; PRESTON, C.A.; MCGOWAN, C.M. Effects of early intensive posoperative physiotherapy on limb function after tibia plateau leveling osteotomy in dogs with deficiency of the cranial cruciate ligament. Am. J. Vet. Res., v.67, p.529-536, 2006.

MURRAY, M.M.; SPINDLER, K.P.; DEVIN, C. et al. Use of collagen-platelet rich plasma scaffold to stimulate healing of a central defect in the canine ACL. J. Orthop. Res., v.24, p.820-830, 2006

MURRAY, M.M.; SPINDLER, K.P.; BALLARD, P. et al. Enhanced histologic repair in a central wound in the anterior cruciate ligament with a collagen-platelet rich plasma scaffold. J. Orthop. Res., v.25, p.10071017, 2007. 
MUZZI, L.A.L.; REZENDE, C.M.F.; MUZZI, R.A.L. Fisioterapia após substituição artroscópica do ligamento cruzado cranial em cães I - avaliação clínica, radiográfica e ultrassonográfica. Arq. Bras. Med. Vet. Zootec., v.61, p.805-814, 2009.

NEUMANN, S.; VIEFHUES, G. Intraartikuläre Injektion von autologem Thrombozytenkonzentratbei der OCD. Veterinärspiegel, v.20, p.22-26, 2010.

PEI, M.; CHEN, D.; LI, J. et al. Histone deacetylase 4 promotes TGF- $\beta 1$-induced synovium-derived stem cell chondrogenesis but inhibits chondrogenically differentiated stem cellhypertrophy. Differentiation, v.78, p.260-268, 2009.
SILVA, R.F.; REZENDE, C.M.F; PAES-LEME, F.O. et al. Evaluación del método del tubo para concentrar plaquetas caninas: estudio celular. Arch. Med. Vet., v.43, p.95-98, 2011.

TRUMBLE, T.N.; BILLINGHURST, R.C.; BENDELE, A.M. et al. Evaluation of changes in vertical ground reaction forces as indicators of meniscal damage after transection of the cranial cruciate ligament in dogs. Am. J. Vet. Res., v.66, p.156-163, 2005.

VASSEUR, P.B. Stifle joint. In: SLATTER, D. Textbook of small animal surgery. $2^{\text {nd }}$ ed. Philadelphia: Elsevier, 2003. p.2090-2133. 